\title{
Study on Paeoniflorin Promoting the Recovery of Motor Function of Rat Spinal Cord Injury by Mediating MAPK/ERK and Akt/mTOR Signal Pathway
}

\author{
Pei Zhang ${ }^{*}$, Nan Wu ${ }^{2}$, Zhijun Song ${ }^{3}$, Zhengfu Tai ${ }^{4}$ \\ ${ }^{1}$ School of Chemical Engineering, Yunnan Open University, Kunming 650500, Yunnan, China \\ ${ }^{2}$ School of Medical, Yunnan University of Business Management, Kunming 650106, Yunnan, China \\ ${ }^{3}$ National Engineering Institute for the Research and Development of Endangered Medicinal Resources in Southwest China, Guangxi \\ Botanical Garden of Medicinal Plants, Nanning 530023, Guangxi, China \\ ${ }^{4}$ Sichuan Kelun Pharmaceutical Research Institute, Chengdu 610500, Sichuan, China \\ Email: 363164339@qq.com
}

\begin{abstract}
Spinal cord injury (SCI) is a devastating disease that can cause severe motor, sensory, and autonomic dysfunction. There is currently no effective treatment. Paeonia lactiflora is a traditional Chinese herbal medicine, which has antispasmodic, analgesic, and blood circulation effects. Paeoniflorin (PEF) is a medicinal plant isolated from Paeoniae Radio, and it is widely used in East Asia. A large number of studies have shown that PEF has a powerful neuroprotective effect. However, the potential mechanism of PEF on SCI needs further study. This project uses Basso Beattie Bresnahan (BBB) motor function score and open field test to evaluate neurological function, and uses immunofluorescence method to detect brain-derived neurotrophic factor (BNDF) and neurotrophin-3 (NT). -3) Protein expression, Western blot is used to detect protein expression level, and RT-PCR is used to detect mRNA expression level. Thus, a rat spinal cord injury model was established to observe the effects of AT/mTOR and MAPK/ERK signal pathways activated by PEF on nerve regeneration and functional recovery in rats with spinal cord injury.
\end{abstract}

Keywords: spinal cord injury, paeoniflorin, recovery

\section{Introduction}

Spinal cord injury (SCI) is a devastating disease that can cause severe motor, sensory, and autonomic dysfunction. There is currently no effective treatment. The average incidence of spinal cord injury worldwide is estimated to be 1:1000, and the average incidence is 4 to 9 cases per 100,000 population per year. The average incidence of spinal cord injury in developing countries is estimated to be $25.5 /$ million/year, ranging from 2.1 to $130.7 /$ million/year. Spinal cord injury has obvious characteristics and can be divided into two mechanisms: primary mechanical injury and secondary injury. Spinal cord injury is considered to be an incurable disease with uneven recovery and uncertain prognosis, but many methods have opened up new directions for the treatment of spinal cord injury, including surgical decompression, neuroprotective agents, and so on.

Paeonia lactiflora is a traditional Chinese herbal medicine, belonging to the Ranunculaceae family. It has antispasmodic, analgesic, and blood-stimulating effects. Paeoniflorin (PEF) is the main medicinal active ingredient in peony. It is a bicyclic monoterpene glycoside compound. It is a medicinal plant isolated from peony (Paeoniae Radio) and has a wide range of applications in East Asia. A large number of studies have shown that PEF has a powerful neuroprotective effect and can improve central nervous system diseases, such as cognitive impairment, depression and Parkinson's.

Studies have shown that the neuroprotective effect of PEF may be related to the anti-inflammatory and anti-apoptotic effects mediated by TLR4/NF-кB. However, the potential mechanism of PEF on SCI needs further study. This project aims to establish a rat spinal cord injury model to observe the effects of AT/mTOR and MAPK/ERK signal pathways activated by $\mathrm{PEF}$ on nerve regeneration and functional recovery in rats with spinal cord injury.

Paeoniflorin is the main active ingredient of the commonly used traditional Chinese medicine Paeonia lactiflora, which belongs to the bicyclic monoterpenoid glycoside compound. In recent years, scholars at home and abroad have carried out more in-depth research on the pharmacological effects of paeoniflorin. They found that paeoniflorin has many functions such as anti-inflammatory, immune regulation and neuroprotection. Its role and potential have been valued by scientific researchers, especially the neuroprotective effect of paeoniflorin is one of the focuses of research in recent years.

In anti-Alzheimer's disease studies, it was found that paeoniflorin can significantly improve the cognitive function of $\mathrm{AD}$ transgenic mice, increase the $\mathrm{Bcl}-2 / \mathrm{Bax}$ ratio and the expression level of $\mathrm{p}$-Akt, and down-regulate the expression of 
p-p38MAPK to reduce inflammation Reaction and caspase-3 activity. Paeoniflorin can also inhibit the neuroinflammation

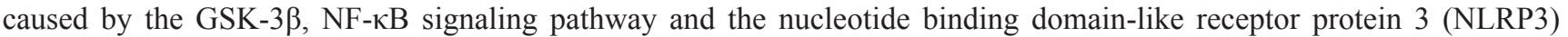
inflammasome, and affect the pathological changes of APP/PS1 transgenic mice. To the effect of anti-inflammatory and anti$A \beta$ deposition. The activation of microglia induced by A $\beta 1-42$ is also one of the pathological changes of AD. Paeoniflorin can pretreatment inhibit $A \beta 1-42$ induced microglia (TNF)- $\alpha$, interleukin(1L)-1 $\beta$ and 1L-6 production; at the same time inhibit the nuclear translocation of NF-кB p65 subunit and IkBa phosphorylation; Reduce the release of CXCL1 and CCL-2; inhibit the increase of vascular endothelial growth factor and its receptor 1 (Flt-1).

Paeoniflorin can also reduce the ischemia-reperfusion injury induced by cerebral infarction in rats. Its pretreatment and post-modeling treatment can reduce the ratio of cerebral infarct areas. Preprocessing can also reduce neurological deficit points. Microvascular ED-1, 1L-1 $\beta$, TNF- $\alpha$, ICAM-1 counts, and the increase of MPO immunopositive cells and apoptotic cells can all be reduced by pretreatment. It can also inhibit the excessive activation of astrocytes, nerve apoptosis, up-regulate NeuN, MAP-2 and Bcl-2, and down-regulate Bax, play an anti-apoptotic effect, thereby reducing cerebral ischemic damage. Paeoniflorin also protects rat brain damage caused by ischemia by inhibiting MAPKs/NF- $\mathrm{KB}-$ mediated inflammatory damage, and can significantly inhibit the excessive activation of astrocytes and microglia caused by transient middle artery ligation (MCAO), And prevent the up-regulation of inflammatory cytokines (TNF- $\alpha, 1 \mathrm{~L}-1 \beta$, iNOS, COX-2 and 5-LOX). Long-term use of PEF can inhibit the activation of JNK and p38MAPK, and increase the activation of ERK, and at the same time can reverse the activation of the NF- $\mathrm{KB}$ signaling pathway caused by ischemia. In the hydrogen peroxide-treated PC12 cell model, paeoniflorin can inhibit the NF- $\kappa$ B pathway, the expression of $1 \mathrm{~L}-1 \beta$ and TNF- $\alpha$ by scavenging oxygen free radicals and reducing the release of lactate dehydrogenase, thereby preventing apoptosis. Anti-oxidant damage.

In summary, as one of the main components of Chinese peony, paeoniflorin (PEF) has a variety of biological activities such as improving blood circulation and immune regulation. It has shown anti-inflammatory and antioxidant effects in a variety of animal models. Inflammation is an important part of secondary injury. A large number of studies have shown that PEF has a powerful neuroprotective effect and plays an important regulatory role in its pathogenesis. The molecular mechanisms involved in the research are often closely related to related inflammatory signaling pathways. Studies have shown that the neuroprotective effect of PEF may be related to the anti-inflammatory and anti-apoptotic effects mediated by TLR4/NF- $\kappa$ B. However, the potential mechanism of PEF on spinal cord injury (SCI) needs further study. Therefore, this article is to establish a rat spinal cord injury model to observe the effects of AT/mTOR and MAPK/ERK signal pathways activated by PEF on nerve regeneration and functional recovery in rats with spinal cord injury.

\section{Experimental process}

\subsection{Animals and groups}

75 male SPF Wistar rats, weighing 250-280 g, were purchased from Kunming Institute of Zoology, Chinese Academy of Sciences. All animals were placed in a constant temperature $\left(22 \pm 1^{\circ} \mathrm{C}\right)$, constant humidity $(55 \% \sim 65 \%)$, ventilated, dry, and quiet environment, alternating day and night for 12 hours, eating and drinking freely, and cleaning the cage every 2 days. The experimental rats were tested after two weeks of acclimatization. All experimental rats were randomly divided into 5 groups of OUPS groups $(\mathrm{n}=15)$ : sham operation group, spinal cord injury model group, SCI+30mg/kg PEF (low dose), SCI+60mg/ $\mathrm{kg}$ PEF (medium dose), SCI $+90 \mathrm{mg} / \mathrm{kg}$ (high dose). Before the experiment started, the study was approved by the Ethics Committee of Yunnan Open University.

\subsection{Establishment of rat model of spinal cord injury}

All rats were anesthetized with sodium pentobarbital. In a sterile environment centered on the T10 spinous process, cut about $2 \mathrm{~cm}$ along the longitudinal line of the spinal cord, excise the muscles on the T10 vertebral body, remove the lamina, and fully expose the spinal cord as the impact area. Except for the sham operation group, the rats in the other groups hit the spinal cord with a weight of $30 \mathrm{~g}$, and then stopped bleeding. After operation, $1 \times 105 \mu \mathrm{L}$ penicillin sodium was injected intraperitoneally to prevent postoperative infection and analgesia. Penicillin $8 \times 104 \mathrm{U}$ was intraperitoneally injected 3 days after operation to prevent infection. Manual urination was continued for 8 hours after the operation until the urination function of the rats recovered automatically. On the 3rd day after operation, PEF was injected intraperitoneally at a low dose of $30 \mathrm{mg} / \mathrm{kg}$, a medium dose of $60 \mathrm{mg} / \mathrm{kg}$, and a high dose of $90 \mathrm{mg} / \mathrm{kg}$.

\subsection{Basso Betty Bresnahan (BBB) scoring and opening test}

Use the blood-brain barrier to check the motor function of the hind limbs. The tests were carried out at $0 \mathrm{~d}, 1 \mathrm{~d}, 7 \mathrm{~d}, 14 \mathrm{~d}$, $21 \mathrm{~d}$ and $28 \mathrm{~d}$. Hind limb motor function is divided into 0-21 points according to the 22-level BBB score. A score of 0 means 
that the hind limbs are incapable of movement, and a score of 21 means that the hind limbs are completely normal.

Field experiments are a method to evaluate the autonomous activities, exploratory behaviors and nervousness of experimental animals in a new environment. The number of grid crossings and the number of uprights can reflect the degree of autonomous activities and limb activities of rats. In the experiment, the rats were placed in a central grid in an open field. After allowing them to acclimate for 2 minutes, the number of grid crossings and the number of erections ED in the following 4 minutes were recorded, and the degree of mobility impairment of the experimental rats was evaluated.

BBB score and open field test results show that PEF can significantly improve the neurological function of SCI rats. BDNF and NT-3 increased in sham group, BDNF and NT-3 increased in SCI group, and BDNF and NT-3 increased in SPF group. The levels of TNF- $\alpha$, IL-1 $\beta$ and il-6 in the SCI group were significantly higher than the false ones, while il-10 was significantly lower, indicating the inflammatory state of SCI rats. After continuous administration of PEF, the relative mRNA expression of ERK and p38 was significantly down-regulated, indicating that PEF can significantly regulate the MAPK/ERK signaling pathway. Compared with the sham group, the expression levels of $\mathrm{p}-\mathrm{AKT} / \mathrm{AKT}, \mathrm{p}-\mathrm{mTOR} / \mathrm{mTOR}$ protein and AKT and mTOR mRNA in the SCI group were significantly reduced. When PEF (60 mg/kg and $90 \mathrm{mg} / \mathrm{kg}) \mathrm{was}$ administered, the levels of these proteins and mRNA increased significantly.

\section{Conclusion}

From this study, PEF can play a role in SCI through MAPK/ERK and Akt/mTOR signaling pathways.

\section{Acknowledgments}

This article was funded by the Yunnan Open University Project Fund. The name of the project is: "Paeoniflorin promotes the recovery of motor function after spinal cord injury in rats" in Yunnan Open University. The topic number is 19 YNOU05.

\section{References}

[1] Zhao H, Chen S, Gao K, et al. Resveratrol Protects Against Spinal Cord Injury by Activating Autophagy and Inhibiting Apoptosis Mediated by the SIRT1/AMPK Signaling Pathway. Neuroscience. 2017; (348): 241-251.

[2] Assinck P, Duncan GJ, Hilton BJ, Plemel JR, Tetzlaff W. Cell transplantation therapy for spinal cord injury. Nat Neurosci. 2017; (20): 637-647, .

[3] Thietje R and Hirschfeld S. Epidemiology of Spinal Cord Injury. In: Norbert Weidner, Rüdiger Rupp, Keith E. Tansey. Neurological Aspects of Spinal Cord Injury. Cham: Springer International Publishing Switzerland; 2017.

[4] Rodrigues LF and Moura-Neto V. Biomarkers in Spinal Cord Injury: from Prognosis to Treatment. Molecular Neurobiology. 2018; 1-13.

[5] Jalan D, Saini N, Zaidi M, Pallottie A, Elkabes S and Heary RF. Effects of early surgical decompression on functional and histological outcomes after severe experimental thoracic spinal cord injury. J Neurosurg Spine. 2017; (26): 62-75.

[6] Vasconcelos NL, Gomes ED, Oliveira EP, et al. Combing neuroprotective agents: effect of riluzole and magnesium in a rat model of thoracic spinal cord injury. Spine Journal Official Journal of the North American Spine Society. 2016; (16): 1015-1024.

[7] Sámano C and Nistri A. Mechanism of Neuroprotection Against Experimental Spinal Cord Injury by Riluzole or Methylprednisolone. Neurochemical Research. 2017; 1-14.

[8] Wu Q, Jing Y, Yuan X, et al. Melatonin treatment protects against acute spinal cord injury-induced disruption of blood spinal cord barrier in mice. Journal of Molecular Neuroscience. 2014; (54): 714-722.

[9] Heo SH, Lee DS, Ham SH, et al. Pharmacokinetic evaluation of paeoniflorin after oral administration of Paeoniae Radix extract powder to healthy Korean subjects using UPLC-MS/MS. Journal of Pharmaceutical Investigation. 2016; (46): 273-282.

[10] Salunga TL, Tabuchi Y, Takasaki I, et al. Identification of genes responsive to paeoniflorin, a heat shock protein-inducing compound, in human leukemia U937 cells. International Journal of Hyperthermia the Official Journal of European Society for Hyperthermic Oncology North American Hyperthermia Group. 2007; (23): 529-537.

[11] Kapoor S. Neuroprotective effects of paeoniflorin: an emerging concept in neurology. Folia Neuropathologica. 2013; (51): 92-92.

[12] Manayi A, Omidpanah S, Barreca D, et al. Neuroprotective effects of paeoniflorin in neurodegenerative diseases of the central nervous system. Phytochemistry Reviews. 2017; (16): 1-9.

[13] Zhang Y, Qiao L, Xu W, et al. Paeoniflorin Attenuates Cerebral Ischemia-Induced Injury by Regulating $\mathrm{Ca}^{2+} / \mathrm{CaMKII}$ CREB Signaling Pathway. Molecules. 2017; (22): 359. 
[14] Feng Z, Sun Y, Liu R, et al. Neuroprotective mechanisms of Paeoniflorin on spinal cord injury in rats. Immunological Journal. 2016.

[15] Wang R, Peng X, Wang L, et al. Preparative purification of peoniflorin and albiflorin from peony rhizome using macroporous resin and medium-pressure liquid chromatography. Journal of Separation Science. 2012; (35): 1985-1992.

[16] Chen T, Guo ZP, Jiao XY, et al. Peoniflorin suppresses tumor necrosis factor- $\alpha$ induced chemokine production in human dermal microvascular endothelial cells by blocking nuclear factor-kB and ERK pathway. Archives of Dermatological Research. 2011; (303): 351-360.

[17] Wang X, Kong K, Shang J. A study on comparing open field test with combined behaviour score after thoracic spinal cord injury in rats. Journal of Shantou University Medical College. 2004; (17): 199-201.

[18] Martinez M, Brezun JM, Bonnier L, Xerri C. A new rating scale for open-field evaluation of behavioral recovery after cervical spinal cord injury in rats. Journal of Neurotrauma. 2009; (26): 1043-1053. 